\title{
Phase transitions in a two parameter model of opinion dynamics with random kinetic exchanges
}

\author{
Parongama $\operatorname{Sen}^{1}$ \\ ${ }^{1}$ Department of Physics, University of Calcutta, 92 Acharya Prafulla Chandra Road, Kolkata 700009, India
}

\begin{abstract}
Recently, a model of opinion formation with kinetic exchanges has been proposed in which a spontaneous symmetry breaking transition was reported [M. Lallouache et al, Phys. Rev. E, 82 056112 (2010)]. We generalise the model to incorporate two parameters, $\lambda$, to represent conviction and $\mu$, to represent the influencing ability of individuals. A phase boundary given by $\lambda=1-\mu / 2$ is obtained separating the symmetric and symmetry broken phases: the effect of the influencing term enhances the possibility of reaching a consensus in the society. The time scale diverges near the phase boundary in a power law manner. The order parameter and the condensate also show power law growth close to the phase boundary albeit with different exponents. The exponents in general change along the phase boundary indicating a non-universality. The relaxation times, however, become constant with increasing system size near the phase boundary indicating the absence of any diverging length scale. Consistently, the fluctuations remain finite but show strong dependence on the trajectory along which it is estimated.
\end{abstract}

PACS numbers: 87.23.Ge, 89.75.Da, 64.60.F-

Various models for opinion formation have been proposed in recent times in which a collective consensus emerges out of individual opinions [1 [5]. Opinion can be assumed to be a discrete or continuous variable and it dynamically evolves according to the interactions between the individuals. The change in the opinion of an individual may be effected by binary interactions or may be dictated by a set of other individuals. The evolution usually leads to a steady state characterised either by a homogeneous state where people have similar opinion or a heterogeneous behaviour where people have widely different opinions. The interactions of the individuals in opinion dynamics models can be studied in terms of appropriate tunable parameters and it is of interest to observe whether such parameters can drive a phase transition in the system [ $6-10]$.

While several different schemes have been proposed for possible evolution of opinions, a number of models have adopted the idea of kinetic exchanges in opinion formation [10-14]. In one such recently introduced model [10], the opinions of individuals, continuously varying from -1 to +1 , were assumed to change after pairwise interactions. A parameter representing "conviction" dictated the way opinions were exchanged. Beyond a threshold value of this parameter, the opinions of the individuals were seen to reach a consensus while below this, all individuals remained in a "neutral" state with their opinions attaining a zero value. Hence the model shows spontaneous symmetry breaking. Such symmetry breaking transitions have also been observed earlier [8, 9], in binary opinion models in which the ordered phase is one in which a consensus is reached. This is a symmetry broken phase while in the disordered phase, the opinions average out to zero. In [10], the so called disordered phase is very special in the sense the opinion of each individual is identically zero making the average equal to zero trivially. Obviously there is no degeneracy in this case and neither any fluctuation. Here, at the transition point, ev- idence of the existence of a diverging timescale was also confirmed.

We introduce in this paper, a generalisation of the above model where a second parameter, representing the "influencing" capability of individuals is incorporated. So individuals are now characterised by two parameters, one which represents one's belief in one's own opinion (conviction) and the other the ability to influence others. In 10], these two qualities were identical. We argue that in general these may be different, e.g., a person with a firm belief might not have the same degree of influencing others. We keep things general allowing both parameters to freely vary from 0 to 1 and investigate the phase transitions in the two dimensional parameter space. Other variations of the basic model have also been considered recently [15.

In the present model, let $x_{i}(t)$ be the opinion of the $i$ th individual at time $t$; then after an interaction of the $i$ th and $j$ th agents, the opinions of the two individuals are changed according to

$$
\begin{gathered}
x_{i}(t+1)=\lambda_{i} x_{i}(t)+\epsilon_{1} \mu_{j} x_{j}(t) \\
x_{j}(t+1)=\lambda_{j} x_{j}(t)+\epsilon_{2} \mu_{i} x_{i}(t),
\end{gathered}
$$

where $\epsilon_{1}$ and $\epsilon_{2}$ are independent random variables ranging from zero to one. Making $\lambda_{i}=\mu_{i}$, one gets back the model of [10]. The opinions of both the individuals are changed at the same time, and the interacting individuals are chosen randomly.

In the simplest picture, we keep the two parameters $\lambda$ and $\mu$ independent of the agents, i.e, assume a homogeneous population having identical $\lambda$ and $\mu$.

The introduction of the parameter $\mu$ different from $\lambda$ lends a different connotation to the original model studied in [10]. One can now interpret the first term containing $\lambda$ as a self interaction term and the term containing $\mu$ representing the influence from others as an interaction term. 
A proper order parameter for this model is $m=$ $\left|\sum_{i} x_{i}\right| / N$ in analogy with magnetic system. Hence in the disordered state, $m=0$. In case of [10], one always ends up with either all zero opinions (in the disordered state) or all positive/negative opinions in the ordered state. This is in contrast with other models of spontaneous symmetry breaking [8, 9] where the disordered state is a mixture of different opinions. However, a disordered state where the average opinion is zero and the individuals have identical opinions can only be possible in models with continuous opinions distributed with both positive and negative values. This so called disordered state with all $x_{i}=0$ can be regarded as a special case of paramagnetic state in the magnetic language. The difference between this state and a general paramagnetic state is that there is no fluctuations here.

Let us first discuss an extreme limiting case of the present model. The aim in this kind of models is to look at the dynamical evolution starting from a completely random state. Interestingly, when $\mu=0$ and for any $\lambda<1, x_{i}$ being less than or equal to 1 in magnitude, will rapidly vanish. However, for $\lambda=1, \mu=0$, opinions will not evolve at all. Hence at this particular point, although $m=0$, the fluctuations remain nonzero in the thermodynamic limit. Such a situation is not possible to realise in the case when $\lambda=\mu$. We will discuss other interesting features of the model close to this point later in the paper.

In the steady state, we get the condition for nonzero solutions of $\left\langle x_{i}\right\rangle$ as,

$$
(1-\lambda)^{2}=\left\langle\epsilon_{1} \epsilon_{2}\right\rangle \mu^{2}
$$

Since $\epsilon_{1}$ and $\epsilon_{2}$ are independent random variables with mean value equal to 0.5 , and as $\lambda, \mu$ cannot exceed 1 , the above condition reduces to

$$
\lambda=1-\mu / 2
$$

We have carried out simulations for a population of $N=2^{m}$ individuals in general (starting from $N=32$ ), the essential results are found to be independent of system size beyond $N=128$. In the simulations with $N$ individuals whose opinions are randomly distributed initially, we first investigate the steady state behaviour and find that indeed, there is a threshold phenomena as the average opinions shows spontaneous symmetry breaking above a phase boundary occurring in the $\lambda-\mu$ plane; the phase boundary obtained numerically matches exactly with (4).

For $\lambda=1$, we find that the final state is not only ordered, but is completely polarised in the sense that the opinions of all the individuals are equal and exactly 1 (or -1 ) for all values of $\mu$ except $\mu=0$ (which we have already discussed). Thus at $\lambda=1, \mu=0$, we have a sharp discontinuity in $m$. So there is a line $\lambda=1$ in the ordered phase where the fluctuations vanish completely. For other values of $\lambda \neq 1$, the nature of the phase is dictated by both $\mu$ and $\lambda$.

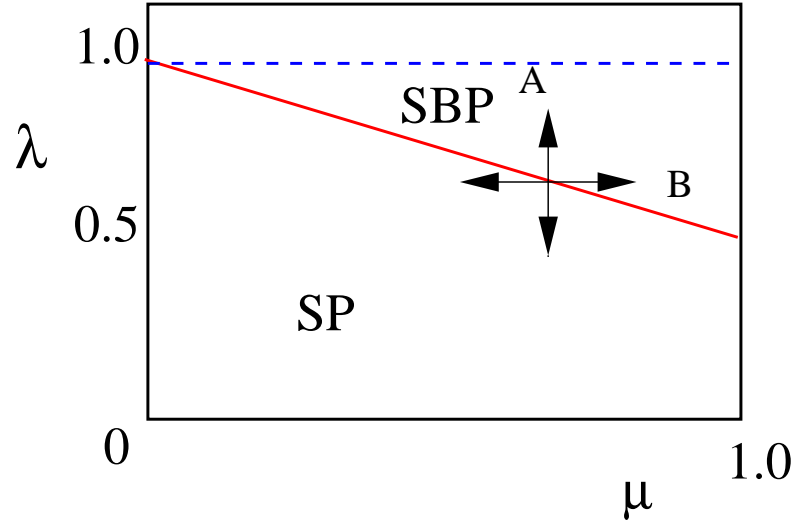

FIG. 1: (Color-online) The phase boundary obtained by numerical simulation coincides exactly with that given in eq (4). SP denotes the symmetric phase and SBP the symmetry broken phase. The paths $\mathrm{A}$ and $\mathrm{B}$ are possible trajectories along which the different studies can be made. Along the dashed line $\lambda=1$, the opinions of all the agents are equal and take extreme values in two possible ways, either $x_{i}=1$ or $x_{i}=-1$ for all $i$.

The implication of the above observation is quite intricate: it shows that in case we have a model society where everyone is fully convinced about one's opinion, the minimum interaction will be able to make the whole population polarised completely and perfectly (i.e., with opinions exactly equal to +1 or -1 ). Since a society without interactions is not conceivable, and that such polarisations seldom take place, it has to be concluded that $\lambda=1$ is indeed an unrealistic idealised value.

The phase diagram is plotted in Fig. 11 We investigate the nature of the transition at different points on the phase boundary. This is done by varying the parameters close to the transition points $\left(\lambda_{c}, \mu_{c}\right)$ on the phase boundary and can be done in several ways in a two dimensional plane. We choose two trajectories: path A, where we keep $\mu$ fixed at $\mu_{c}$ and vary $\lambda$, and path $\mathrm{B}$, where $\lambda$ is fixed at $\lambda_{c}$ and $\mu$ is varied. In some special cases, all possible trajectories cannot be explored, e.g., for $\lambda_{c}=1, \mu_{c}=0$, the path $\mathrm{A}$ does not exist.

Unless explicitly mentioned, the initial state is always taken to be completely random. The static and dynamical results along both patha $\mathrm{A}$ and $\mathrm{B}$ have been obtained and compared. To check the divergence of timescales close to the transition point, we find that the order parameter given by the average opinion denoted by $m=\left|\sum_{i} x_{i}\right| / N$ behaves conventionally as

$$
m(t) \propto \exp (-t / \tau)
$$

in the symmetric or disordered phase where $m(t \rightarrow \infty)=$ 0 , and as

$$
m=m_{0}\left(1-e^{-t / \tau}\right)
$$

in the ordered or symmetry broken phase. In the ordered phase, $m_{0}$, the equilibrium value attained at large times, 

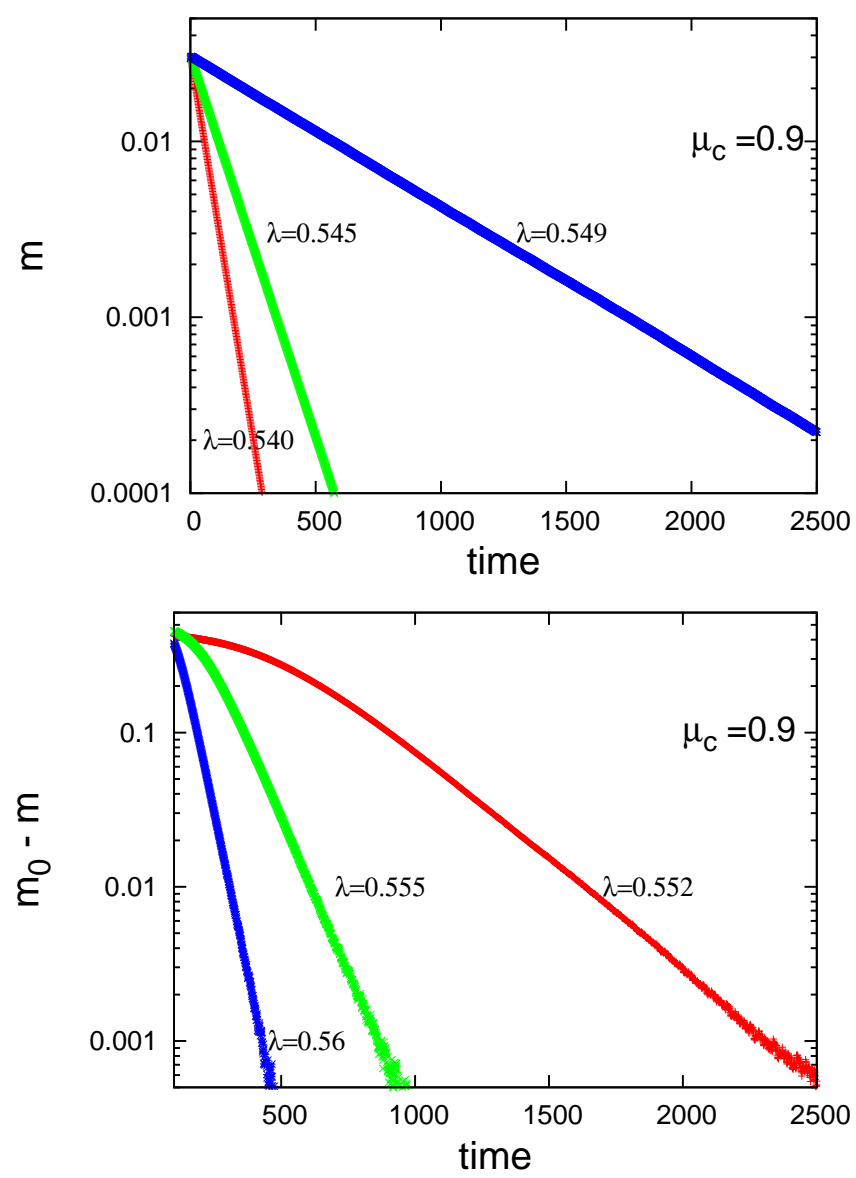

FIG. 2: (Color-online) The behaviour of the order parameter along path $\mathrm{A}$ for $\lambda\left\langle\lambda_{c}\right.$ and $\lambda>\lambda_{c}$ are shown for a system of $N=256$.

is estimated by averaging $m(t)$ over the last few hundred time steps.

Results for path $A$ :

In Fig. 2, we plot $m(t)$ in the disordered phase and $m_{0}-m(t)$ in the ordered phase to get the exponential decay in both cases. Estimating $\tau$ from the slopes of these curves, we show the variation of $\tau$ with $\left|\lambda-\lambda_{c}\right|$ at different locations on the phase boundary given by $\lambda_{c}, \mu_{c}$ in Fig 3. (The locations are indicated by the values of $\mu_{c}$ only as $\lambda_{c}$ is related to $\mu_{c}$ and the latter is kept constant.) There is a power law variation:

$$
\tau \propto\left(\lambda-\lambda_{c}\right)^{-\rho}
$$

which is true for both $\lambda\left\langle\lambda_{c}\right.$ and $\lambda>\lambda_{c}$ with same values of $\rho$.

The value of the exponent $\rho$ changes very slowly along the phase boundary indicating a non-universal behaviour. $\rho$ varies systematically as $\mu_{c}$ is increased; e.g., for $\mu_{c}=0.4, \rho=1.04 \pm 0.01$, for $\mu_{c}=2 / 3, \rho=1.10 \pm 0.03$ while for $\mu_{c}=0.9, \rho=1.21 \pm 0.01$.

To check the effect of finite sizes on the time scales, we estimate $\tau$ very close to the phase boundary for different system sizes. Plotted in the inset of Fig. 3, the

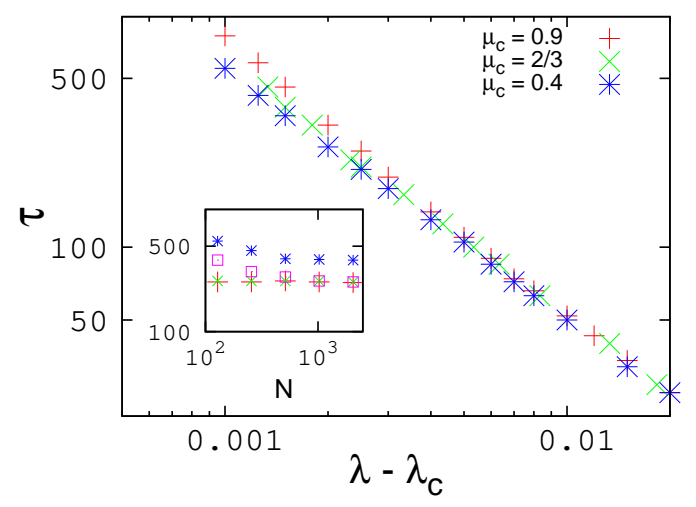

FIG. 3: (Color-online) The timescale variation along path $\mathrm{A}$ is shown for three different values of $\mu_{c}$ for $N=256$ for the order parameter $m$. Inset shows the values of $\tau$ against the system size $N$ for $\lambda-\lambda_{c}=0.013\left(^{*}\right)$ and $\lambda-\lambda_{c}=0.02$ (other symbols)

result shows an interesting variation. The timescales are larger for smaller system sizes and converge for larger sizes. We conclude that the timescales are independent of the system size as it increases.

We have studied the behaviour of some other quantities close to the phase transition point also. The equilibrium value of the order parameter $m$ shows a power law behaviour with $\left(\lambda-\lambda_{c}\right)$ for $\lambda>\lambda_{c}$,

$$
m \propto\left(\lambda-\lambda_{c}\right)^{\beta}
$$

where $\beta$ is quite strongly dependent on the point on the phase boundary, e.g., $\beta=0.079 \pm 0.001$ at $\mu_{c}=0.4$ and $\beta=0.155 \pm 0.001$ at $\mu_{c}=0.9$. This result again supports the claim that the phase transition is non-universal.

Another interesting quantity in this type of models is $p$, the so called condensate fraction, defined as the fraction of individuals having extreme opinions \pm 1 . We find that it shows a behaviour similar to $m$, i.e., it is zero below the critical point and finite above it. The equilibrium values show scaling with $\lambda-\lambda_{c}$ for $\lambda>\lambda_{c}$ with an associated exponent $\beta_{p} \neq \beta$. Once again the values of $\beta_{p}$ show nonuniversality but the nonuniversality is much weaker compared to that found for $\beta$ (see Fig. (5)). For example, $\beta_{p} \simeq 0.91$ for $\mu_{c}=0.4, \simeq 0.95$ for $\mu_{c}=2 / 3$ (this value agrees with another estimate [15]) and $\simeq 1.0$ for $\mu=0.9$.

The time periods $\tau_{p}$ also show scaling with $\lambda-\lambda_{c}$ as shown in Fig 6] The values of the corresponding exponent $\rho_{p}$ are very close to $\rho$ and again has weak nonuniversality. These two exponents appeared to be quite different 


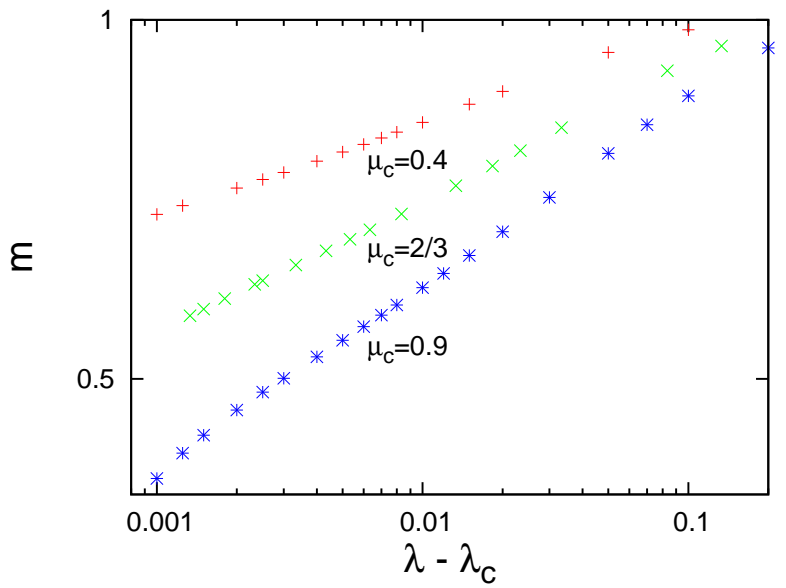

FIG. 4: (Color-online) The equilibrium value of order parameter as a function of $\lambda-\lambda_{c}$ is shown for different values of $\mu_{c}$ along path A for $N=256$. The exponent is seen to be appreciably dependent on $\mu_{c}$.

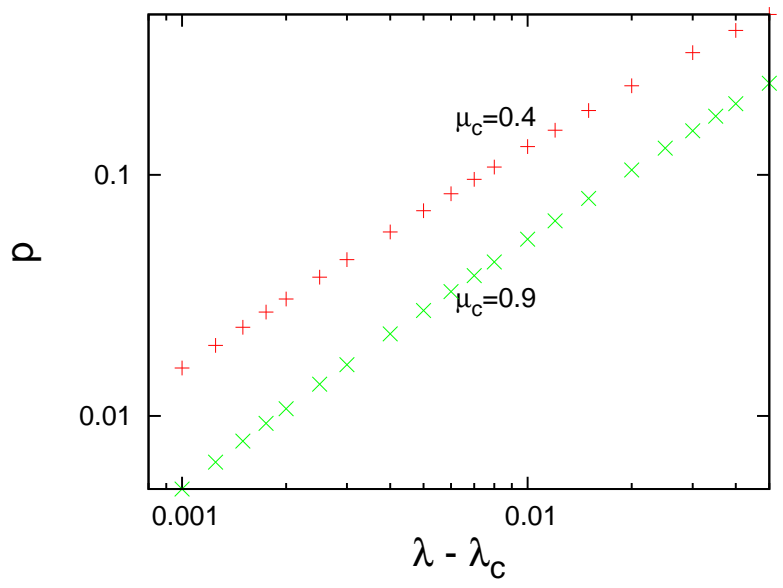

FIG. 5: (Color-online) The equilibrium value of the condensate fraction $p$ as a function of $\lambda-\lambda_{c}$ is shown for two different values of $\mu_{c}$ along path A for $N=256$. The exponent is seen to be weakly dependent on $\mu_{c}$.

in [10]. The values of these exponents depend very sensitively on the range of fitting, choice of $\lambda_{c}, \mu_{c}$ etc. We have taken $\lambda_{c}$ to be that given by (4) for a given $\mu_{c}$ and considered the range $\left|\lambda-\lambda_{c}\right| \leq 0.01$. We have also checked that the $\rho$ and $\rho_{p}$ values become closer as system size is increased. An independent estimate [16] for $\rho_{p}$ for the original model $\left(\lambda_{c}=\mu_{c}=2 / 3\right)$ is found to be $\sim 1.1$ which also agrees with our estimate of $\rho_{p}=1.16 \pm 0.03$ at that point. The fact that $\rho$ and $\rho_{p}$ are alomost equal indicates that there is only one timescale in the system.

One can calculate the fluctuations in the order parameter, $\sigma=\sqrt{\sum_{i} x_{i}{ }^{2} / N-\left(\sum_{i} x_{i} / N\right)^{2}}$, in the steady state. In the symmetric phase, it is zero identically as $x_{i}=0$ for all $i$. The results show that it does not diverge as the phase transition point is approached in the symmetry broken phase. There does appear a peak in the fluctua-

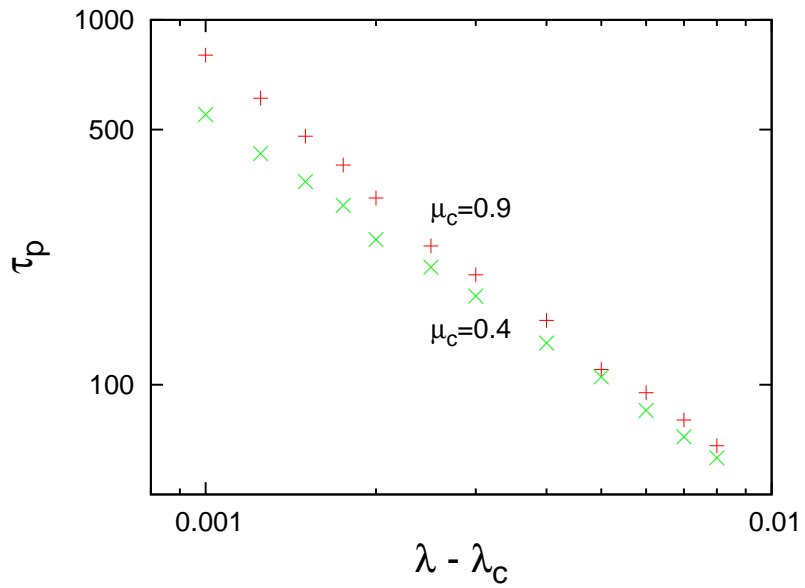

FIG. 6: (Color-online) The timescale variation along path A is shown for two different values of $\lambda_{c}$ for system size $N=256$ for the condensate fraction $p$.

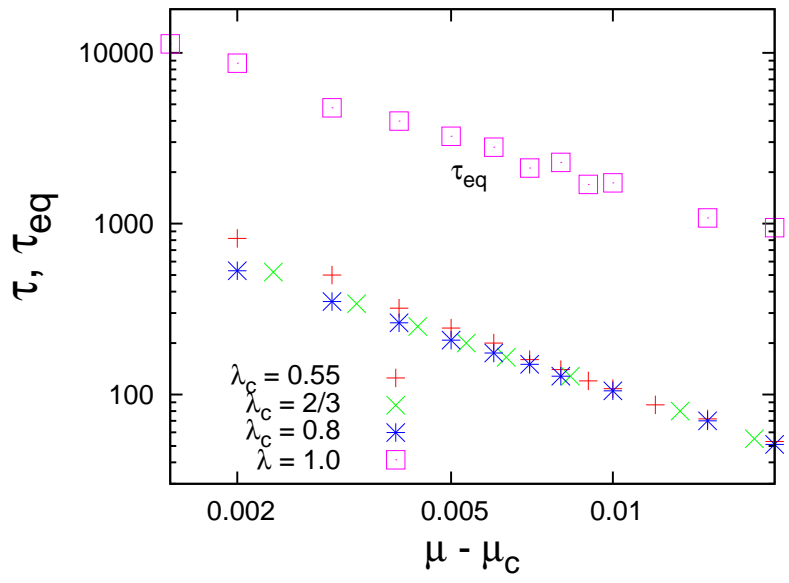

FIG. 7: (Color-online) The timescale $(\tau)$ variation along path $\mathrm{B}$ is shown for three different values of $\lambda_{c}$ for system size $N=256$ for $m ; \tau_{e q}$ is the actual time to equilibriate for $\lambda=1$, for which $\mu_{c}$ is zero.

tion, as in [10], however, there is no power law divergence with $\left(\lambda-\lambda_{c}\right)$. In fact the peak moves away from the transition point for increasing values of $\mu_{c}$ and hence the behaviour of the fluctuations is quite unimportant.

The facts that the timescales are independent of the system size and the fluctuations do not diverge indicate that there is no diverging length scale in the system. That is perhaps because there is no inherent length scales in the system, any individual can interact with any other and thus the model is mean-field like where fluctuations can be ignored.

Results along path $B$ :

Now $\lambda$ is kept constant, i.e., $\lambda=\lambda_{c}$ and $\mu$ is varied close to the phase boundary. The magnitude of the time scales are about twice compared to those in path B along this path, although the value of the exponents are very close. 
Along path $\mathrm{B}$, it is possible to study the behaviour close to the special point $\lambda=1, \mu=0$. At this point, there is no order and no dynamics. As $\mu$ is switched on, the system relaxes to the steady state where the opinion of all individuals are 1 or -1 and it is possible to calculate the time to reach the steady state. This time, $\tau_{e q}$, is found to be one order of magnitude higher than $\tau$ or $\tau_{p}$, and shows a scaling behaviour with $\lambda$ with exponent $0.96 \pm 0.03$. The results for $\tau$ and $\tau_{e q}$ along path $\mathrm{B}$ are shown in Fig 7.

For any quantity $\phi$, one can assume that

$$
\phi \propto\left(\lambda-\lambda_{c}\right)^{a} \Phi\left[\frac{\lambda-\lambda_{c}}{\left(\mu-\mu_{c}\right)^{y}}\right],
$$

where $a$ is the exponent obtained in path $\mathrm{A}$ and the exponent $b$ from path $\mathrm{B}$ is given by $b=a y$. The estimate of $y$ from the timescale $\tau$ turns out to be marginally higher than 1 while that from $m$ is around 0.9 and is not dependent on the critical values of $\mu_{c}$ and $\lambda_{c}$. We conclude that $y$ is a universal exponent with a value close to 1 .

The fluctuations, as in path A, once again show a peak which is further away from the transition point and remain finite even as $\mu \rightarrow 1$, e.g., for $\lambda_{c}=0.55$. This is a major difference with path $\mathrm{A}$, where the fluctuations become zero far away from the phase boundary at $\lambda=1$. The fluctuations for both paths are plotted in Fig 8 .

The fluctuations in $p$ can also be calculated as it varies from configuration to configuration. The results are exactly similar to that of $m$.

So far, we discussed the dynamics when the initial configuration is completely random. In case one studies the nonequilibrium relaxation behaviour starting from a partially/fully ordered state, a power law decay of the order parameter is expected exactly at the critical point. This exponent, however, is not an independent one as it is given by $\beta / \rho$ (for $m$ ) or $\beta_{p} / \rho_{p}$ (for $p$ ). We have checked that for both $m$ and $p$ power law decays with exponents consistent with the already obtained values of $\beta \quad\left(\beta_{p}\right)$ and $\rho\left(\rho_{p}\right)$ can be observed.

In summary, we have studied a model of opinion formation with kinetic exchanges, in which two realistic parameters are used. A phase boundary is obtained in the parameter space. There are two absorbing phases: the symmetric phase and the extreme boundary line $\lambda=1$. The model is a generalisation of a recently proposed model with a single parameter representing conviction in one's opinion. While conviction is expected to restore one's opinion, the influence of others may try to change it. Hence these two factors appear in the dynamical evolution with a randomness represented by $\epsilon$. The ability to influence need not be identical to one's conviction. Representing the two qualities of conviction and influencing as independent parameters, we have shown that the effect of the influencing term is like an interaction which enhances the possibility of reaching a consensus: cooperative interaction is reponsible for any order to exist. The results show that the behaviour of this model with continuous variation of opinions is comaparable to binary
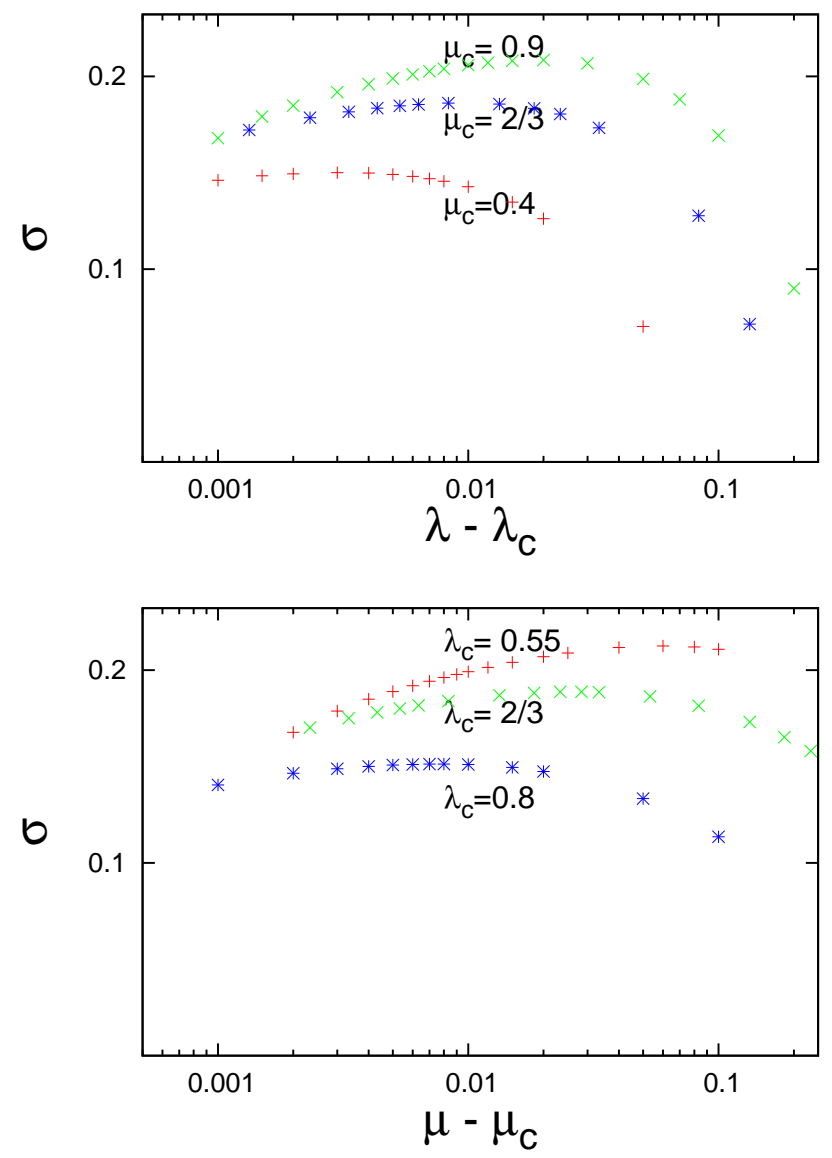

FIG. 8: (Color-online) The equilibrium fluctuations in the order parameter shows no divergence for either path A (top) or B (bottom). The results are shown for $N=256$.

opinion models only at a special point where there is coexistence of many opinions but unlike that in [8] , it is an absorbing state. In fact the entire disordered phase is an absorbing phase in contrast to the binary opinion models.

The phase transition along the boundary is shown to be non-universal as the exponents have different values at different points on the boundary. The nonunivrsality is maximum for the order parameter $m$, while for the other quantities it is weak. The actual values indicate that with larger values of $\mu$, consensus is reached in lesser timescales. It is still not very clear why the nonuniversal behaviour is there but it perhaps indicates that the phase boundary is actually a line of critical points.

Acknowledgments: The author is grateful to Soumyajyoti Biswas, Anjan Chandra and Arnab Chatterjee for very useful discussions and for sharing their results prior to publication and to Abdus Salam ICTP for hospitality where a major part of the work was done. She also thanks S M Bhattacharjee for some comments. Financial support from UPE project is acknowledged. 
[1] D. Stauffer, in Encyclopedia of Complexity and Systems Science edited by R. A. Meyers (Springer, New York, 2009).

[2] C. Castellano, S. Fortunato and V. Loreto, Rev. of Mod. Phys. 81, 591-646 (2009).

[3] T. M. Liggett Interacting Particle Systems: Contact, Voter and Exclusion Processes (Springer-Verlag Berlin 1999).

[4] K. Sznajd-Weron and J. Sznajd, Int. J. Mod. Phys C 11, 1157 (2000);

[5] S. Galam, Int. J. Mod. Phys. C 19, 409 (2008);

[6] A. Baronchelli, L. Dall'Asta, A. Barrat, and V. Loreto, Phys. Rev. E 76, 051102 (2007); C. Castellano, M. Marsili and A. Vespignani, Phys. Rev. Lett. 85, 3536 (2000).

[7] S. Biswas and P. Sen, Phys. Rev. E 80, 027101 (2009); P. Sen, Phys. Rev E 81, 032103 (2010); S. Biswas and P.
Sen, arXiv 1004.3861.

[8] S. Galam, Physica A 333453 (2004).

[9] S. Galam, Surophys. Lett. 70705 (2005).

[10] M. Lallouache, A. S. Chakrabarti, A. Chakraborti, B. K. Chakrabarti, Phys. Rev. E 82056112 (2010).

[11] R. Hegselman, U. Krause, J. Artif. Soc Simul., 5, 2 (2002).

[12] G. Deffuant, N. Neau, F. Amblard and G. Weisbuch, Adv. Complex Sys. 3, 87 (2000).

[13] S. Fortunato, Int. J. Mod. Phys. C 16, 17 (2005).

[14] G. Toscani, Comm. Math. Sc. 4, 481 (2006).

[15] S. Biswas, A. K. Chnadra and A. Chatterjee, arXiv 1010.3190.

[16] S. Biswas, A. K. Chnadra and A. Chatterjee, private communication. 